\title{
Enhancement of Antioxidant Defense System by Epigallocatechin-3- gallate during Bleomycin Induced Experimental Pulmonary Fibrosis
}

\author{
Narayanan SRIRAm, Srinivasan Kalayarasan, and Ganapasam SudHANDIRAN* \\ Department of Biochemistry, University of Madras; Guindy Campus, Chennai-600 025, Tamilnadu, India. \\ Received December 16, 2007; accepted April 15, 2008; published online April 17, 2008
}

\begin{abstract}
Oxidative stress resulting from an imbalance between radical-generating and radical scavenging systems plays an important role in the pathogenesis of pulmonary fibrosis. Epigallocatechin-3-gallate (EGCG), a polyphenol and a major component of green tea, possess a potent antioxidant property. This study was designed to evaluate the potential antioxidative activity of EGCG in the plasma and lungs during bleomycin induced experimental pulmonary fibrosis. Intratracheal administration of bleomycin $(6.5 \mathrm{U} / \mathrm{kg}$ body weight) to rats resulted in significant reduction of body weight, enzymic antioxidants (superoxide dismutase, catalase, glutathione peroxidase and glutathione reductase) and non-enzymic antioxidants (reduced glutathione, vitamin $\mathbf{C}$, vitamin $\mathbf{E}$ and vitamin A). Elevations in lung W/D (wet weight/dry weight) ratio, hydroxyproline content was observed with a synchronized increase in lipid peroxidation markers (thiobarbituric acid reactive substances and hydroperoxides). Intraperitoneal administration of EGCG at a dose of $20 \mathrm{mg} / \mathrm{kg}$ body weight significantly improved the body weight, enzymic and non enzymic antioxidants and considerably decreased the W/D ratio, hydroxyproline and lipid peroxidation marker levels. Histological observations also correlated with the biochemical parameters. Thus, this study confirms the beneficial use of EGCG in alleviating the oxidative stress induced during pulmonary fibrosis.
\end{abstract}

Key words epigallocatechin gallate; bleomycin; lipid peroxidation; flavonoid; pulmonary fibrosis; antioxidant

Superoxide and hydroxyl radicals along with hydrogen peroxide $\left(\mathrm{H}_{2} \mathrm{O}_{2}\right)$ are commonly termed as reactive oxygen species (ROS). The main source of generation of ROS in cells are various metabolic reactions including the incomplete reduction of oxygen in mitochondrial electron transport chain during respiration. ${ }^{1,2)}$ If superoxide radicals are not neutralized, they in turn generate hydroxyl radicals by reacting with $\mathrm{H}_{2} \mathrm{O}_{2}$ in the presence of free iron by classical Fenton reaction. ${ }^{2,3)}$ Thus generated ROS, if not quickly disposed from cellular environment, can drive the cell to a prooxidant state, referred as oxidative stress, by attacking almost all major biological molecules including membrane lipids. ${ }^{2}$ Thwarting the damage inflicted by free radicals and reactive species is the function of a complex antioxidative defense system. This system includes enzymes such as superoxide dismutase (SOD), catalase (CAT) and glutathione peroxidase (GPx) and some of the most commonly used and experimentally studied nonenzymatic antioxidants such as vitamin $\mathrm{E}$, vitamin $\mathrm{C}$, carotenoids and glutathione. ${ }^{4,5)}$ Under some pathological conditions where ROS are formed in excess, the antioxidant systems of cells cannot withstand the oxidative stress and eventually results in damage of cells and tissues. ${ }^{6}$

The lung, as the organ exposed to the highest oxygen concentration, is particularly at risk to the toxic effects of oxygen. ${ }^{7)}$ Several lung diseases have been associated with oxidative stress and linked to oxidant insults. ${ }^{8)}$ Therefore the lungs and tissues of the respiratory tract require a specific defense system against oxidants and free radicals. Pulmonary fibrosis is a pathological state that is characterized by superfluous synthesis and deposition of extracellular matrix (ECM) in the distal airspace, and is probably initiated by acute or chronic lung injury. ${ }^{9,10)}$ The current treatment to pulmonary fibrosis involves corticosteroids, antifibrotic and immunosuppressive agents, with very less achievement. ${ }^{11,12)}$ Therefore, treating pulmonary fibrosis successfully remains an uphill task, and development of drug regimens for the same remains an im- perative challenge.

Bleomycin is an antibiotic used as an anti-cancer drug that has shown efficacy in an array of tumors both in humans and animals. ${ }^{13)}$ The therapeutic utility of the drug has, however, been precluded by severe toxicities especially of the lung. ${ }^{14)}$ Pulmonary fibrosis is the major side effect that is elicited by bleomycin and depends upon the duration and the dosage of the anti-neoplastic drug. Although the exact mechanism of development of pulmonary fibrosis is not yet fully explored, it is believed that bleomycin generates ROS and initiates inflammatory and fibroproliferative changes via a concerted action of various cytokines leading to collagen accumulation in the lung. ${ }^{15,16)}$ Bleomycin induction also results in depletion of endogenous antioxidant defenses thereby increasing the risk of oxidant mediated tissue injury. ${ }^{17,18)}$ Bleomycin induced pulmonary fibrosis in rodents is popular and has been used as a surrogate model for human lung fibrosis. ${ }^{19)}$

Flavonoids are a large group of phenylchromones widely distributed in plant structures. ${ }^{20)}$ They are important in human diet and are present in plant extracts that have been used for centuries in oriental medicine. The pharmacological and biochemical properties of flavonoids have now been extensively reviewed. Antioxidant properties, ROS scavenging, and cell function modulation of flavonoids could account for the large part of their pharmacological activity. The green tea polyphenols are natural plant flavonoids that comprise many types of catechins. Among these, (-)-epigallocatechin-3-gallate (EGCG) is the major polyphenol component of green tea and it is primarily responsible for the beneficiary effects of green tea. Substantial evidence suggests that EGCG elicit anti-oxidant properties by attenuating the lipid peroxidation (LPO) caused by various forms of free radicals. ${ }^{21)}$ The potential antioxidant property of EGCG is attributed to its structure that contains two triphenolic groups. ${ }^{22)}$ Since EGCG has been reported as a better antioxidant than vitamin $\mathrm{E}$ and/or vitamin $C,{ }^{23,24)}$ the present study was aimed to investigate the 
effect of EGCG on antioxidant defense systems during bleomycin-induced pulmonary fibrosis in Wistar rats.

\section{MATERIALS AND METHODS}

Chemicals Bleomycin sulfate was purchased from Sigma chemicals, St. Louis, U.S.A. EGCG was procured from Cayman chemicals, U.S.A. All other chemicals used were of analytical grade.

Animals Male albino Wistar rats, weighing about 180 $210 \mathrm{~g}$, were used in this study. The rats were housed in individual cages and acclimatized for a period of $7 \mathrm{~d}$ before the experiment was conducted. The rats were maintained on standard chow diet (M/s. Hindustan Lever Ltd., Bangalore, India) and were allowed free access to water. The experiments were designed and conducted according to ethical norms approved by the Ministry of Social Justices and Empowerment, Government of India and Institutional Animal Ethics Committee Guidelines (Approval no. 360/01/A/CPCSEA).

Induction of Pulmonary Fibrosis An animal model of bleomycin induced pulmonary fibrosis was induced in rats as previously described, ${ }^{25)}$ with little modifications. For induction of pulmonary fibrosis, male Wistar rats $(n=6)$ received a single dose of $6.5 \mathrm{U} / \mathrm{kg}$ (based on body weight) bleomycin sulfate dissolved in $0.3 \mathrm{ml} 0.9 \% \mathrm{NaCl}$ solution by intratracheal instillation on day 1 of the experimental period. Control rats were given a single intratracheal dose of saline alone.

Experimental Design A pilot study was conducted with five different doses of EGCG $(10,15,20,25,30 \mathrm{mg} / \mathrm{kg}$ body weight), post-treated $6 \mathrm{~h}$ after bleomycin induction and administered intraperitoneally for $28 \mathrm{~d}$ to determine the dosedependent effect in bleomycin-induced rats. It was observed that EGCG treatment at a dose of $20 \mathrm{mg} / \mathrm{kg}$ body weight, significantly $(p<0.05)$ altered bronchoalveolar lavage fluid (BALF) hydroxyproline level and serum activities of alkaline phosphatase (ALP) and lactate dehydrogenase (LDH) to near normal values in bleomycin induced rats after $28 \mathrm{~d}$ of experimental study. Hence, the dose of $20 \mathrm{mg} / \mathrm{kg}$ was chosen for our study.

The rats were divided into four groups (Six rats in each group) as follows,

Group I: Normal control rats

Group II: Normal rats treated with EGCG, intraperitoneally at a dosage of $20 \mathrm{mg} / \mathrm{kg}$ body weight (dissolved in saline, $2 \mathrm{ml} / \mathrm{kg}$ body weight), once daily throughout the experimental period $(28 \mathrm{~d})$

Group III: Bleomycin induced rats

Group IV: Six hours after the bleomycin induction, rats were intraperitoneally treated with EGCG with the concentration and duration as mentioned for group II

After $28 \mathrm{~d}$ of the experimental period, the rats of all the groups were sacrificed by cervical decapitation. Blood was collected in tubes containing potassium oxalate and sodium fluoride, from which plasma was separated.

Wet to Dry Lung Weight Ratio After sacrificing the animals, the lungs were immediately excised and weighed Then they were dried at $50^{\circ} \mathrm{C}$ for $72 \mathrm{~h}$, and weighed again. The wet dry (W/D) lung weight ratio was calculated as an in- dicator of pulmonary edema.

Histological Examination The lung tissue samples were fixed in $10 \%$ buffered formalin, routinely processed and embedded in paraffin. Three-micrometer-thick sections were placed on slides and stained with hematoxylin and eosin (H\&E) and Massons's trichrome. The slides were then evaluated under light microscope (Nikon XDS-1B).

Preparation of Lung Tissue The lung was excised, rinsed in ice-cold physiological saline and homogenized in $0.1 \mathrm{M}$ Tris- $\mathrm{HCl}$ buffer ( $\mathrm{pH}$ 7.4), using a tissue homogenizer with a teflon pestle at $4{ }^{\circ} \mathrm{C}$. The resultant tissue homogenate was used for biochemical measurements.

Biochemical Measurements LPO was determined in the lung tissue homogenate and plasma by measuring the formation of thiobarbituric acid reactive substances (TBARS) according to the method of Ohkawa et al. ${ }^{26)}$ Hydroperoxides were estimated both in plasma and in tissue homogenate by the method described by Jiang et $a .^{27)}$ Enzymic antioxidants were measured in the lung tissues. SOD was measured by the method described by Misra and Fridovich. ${ }^{28)}$ CAT and GPx were measured according to Takahara et al. ${ }^{29)}$ and Rotruck et $a l .{ }^{30)}$ respectively. Glutathione reductase (GR) was analyzed by the method of Staal et al. ${ }^{31)}$

Reduced glutathione (GSH) was measured in plasma and in tissue homogenate by the method of Ellman. ${ }^{32)}$ Vitamin $\mathrm{C}$ was estimated in lungs by the method described by Omaye et $a l^{33)}$ Vitamin E was measured in tissue homogenate according to the method of Desai. ${ }^{34)}$ Vitamin A was estimated in lung homogenate by the method of Bayfield and Cole. ${ }^{35)}$ The total collagen content was estimated by assaying the hydroxyproline content in lung tissue. ${ }^{36)}$ The total protein concentration in the tissue homogenate was estimated by the method of Lowry et al. ${ }^{37)}$

Statistical Methods All the grouped data was evaluated using SPSS/10.0 software. Hypothesis testing method included one-way analysis of variance (ANOVA) followed by least significant difference (LSD) test. $p<0.05$ was considered to indicate statistical significance. All the results were expressed as mean \pm S.D. for six rats in each group.

\section{RESULTS}

Table 1 shows the body weight, lung W/D ratio and hydroxyproline level of control and experimental group of rats. In bleomycin-induced rats, it was found that there is a significant $(p<0.05)$ decrease in the final body weight when compared to control rats. Supplementation of EGCG to bleomycin-induced animals resulted a significant increase in final body weight when compared to bleomycin-induced animals. A significant elevation in lung W/D ratio and hydroxyproline level was evident in bleomycin-induced animals as compared to control animals. Whereas, the lung W/D ratio and hydroxyproline level were lowered significantly in bleomycin-induced animals treated with EGCG.

Table 2 depicts the levels of TBARS and hydroperoxides in the plasma and lungs of control and experimental group of rats. A remarkable elevation in the levels of TBARS and hydroperoxides was observed in the bleomycin-induced rats when compared to control rats. Treatment with EGCG resulted in a significant decrease of these levels as compared to bleomycin-induced animals. 
Table 1. Effect of EGCG on Bodyweight, Lung W/D Ratio and Lung Hydroxyproline Content of Control and Bleomycin Induced Rats

\begin{tabular}{llll}
\hline \hline Experimental groups & $\begin{array}{c}\text { Initial body } \\
\text { weight }(\mathrm{g})\end{array}$ & $\begin{array}{c}\text { Final body } \\
\text { weight }(\mathrm{g})\end{array}$ & $\begin{array}{c}\text { Lung W/D } \\
\text { ratio }\end{array}$ \\
\hline Control & $197 \pm 8.27$ & $226 \pm 9.49$ & $3.78 \pm 0.15$ \\
EGCG & $201 \pm 8.84$ & $233 \pm 10.08$ & $3.80 \pm 0.16$ \\
Bleomycin & $203 \pm 8.72$ & $182 \pm 9.82^{a)}$ & $5.82 \pm 0.33^{a)}$ \\
Bleomycin+EGCG & $198 \pm 8.31$ & $219 \pm 9.85^{b}$ & $4.54 \pm 0.20^{b)}$ \\
\hline
\end{tabular}

Values are given as mean \pm S.D. for groups of 6 rats each. Values are given statistically significant at $p<0.05: a$ ) bleomycin induced $v s$. control; $b$ ) bleomycin + EGCG $v s$. bleomycin induced.

Table 2. Effect of EGCG on Thiobarbituric Acid Reactive Substances (TBARS) and Hydroperoxides in Plasma and Lungs of Control and Bleomycin Induced Rats

\begin{tabular}{|c|c|c|c|c|}
\hline \multirow[b]{2}{*}{ Experimental groups } & \multicolumn{2}{|c|}{ Plasma } & \multicolumn{2}{|c|}{ Lungs } \\
\hline & $\begin{array}{l}\text { TBARS } \\
(\mathrm{nmol} / \mathrm{ml})\end{array}$ & $\begin{array}{l}\text { Hydroperoxides } \\
\left(10^{-5} \mathrm{mmol} / \mathrm{dl}\right)\end{array}$ & $\begin{array}{c}\text { TBARS } \\
\text { (mmol/100 } \mathrm{g} \text { tissue })\end{array}$ & $\begin{array}{l}\text { Hydroperoxides } \\
\text { (mmol/100 g tissue) }\end{array}$ \\
\hline Control & $3.16 \pm 0.13$ & $8.53 \pm 0.52$ & $37.43 \pm 2.28$ & $9.31 \pm 0.58$ \\
\hline EGCG & $3.17 \pm 0.15$ & $8.61 \pm 0.54$ & $36.78 \pm 2.31$ & $9.43 \pm 0.58$ \\
\hline Bleomycin & $7.41 \pm 0.41^{a)}$ & $13.76 \pm 1.03^{a)}$ & $67.86 \pm 5.29^{a)}$ & $20.07 \pm 1.54^{a)}$ \\
\hline Bleomycin+EGCG & $3.49 \pm 0.15^{b)}$ & $10.28 \pm 0.65^{b)}$ & $48.27 \pm 3.37^{b)}$ & $11.94 \pm 0.81^{b)}$ \\
\hline
\end{tabular}

Values are given as mean \pm S.D. for groups of 6 rats each. Values are given statistically significant at $p<0.05: a$ ) bleomycin induced $v s$. control; $b$ ) bleomycin + EGCG $v s$. bleomycin induced.

Table 3. Effect of EGCG on the Activity of Lung Enzymic Antioxidants of Control and Bleomycin Induced Rats

\begin{tabular}{|c|c|c|c|c|}
\hline Experimental groups & SOD & CAT & GPx & GR \\
\hline Control & $4.27 \pm 0.27$ & $18.41 \pm 1.52$ & $2.09 \pm 0.13$ & $1.83 \pm 0.11$ \\
\hline EGCG & $4.21 \pm 0.28$ & $19.06 \pm 1.58$ & $2.04 \pm 0.13$ & $1.89 \pm 0.11$ \\
\hline Bleomycin & $2.94 \pm 0.23^{a)}$ & $11.27 \pm 1.09^{a)}$ & $1.42 \pm 0.11^{a)}$ & $0.86 \pm 0.06^{a)}$ \\
\hline Bleomycin+EGCG & $4.03 \pm 0.26^{b)}$ & $17.13 \pm 1.42^{b)}$ & $1.89 \pm 0.12^{b)}$ & $1.57 \pm 0.10^{b)}$ \\
\hline
\end{tabular}

Units-SOD: $50 \%$ inhibition of adrenaline auto oxidation $/ \mathrm{min}$; CAT: $\mu \mathrm{M} \mathrm{H}_{2} \mathrm{O}_{2}$ Consumed /mg protein/min; GPx: $\mu \mathrm{g}$ GSH utilized/mg protein/min; GR: nmol NADPH oxidized $/ \mathrm{mg}$ protein $/ \mathrm{min}$. Values are given as mean \pm S.D. for groups of 6 rats each. Values are given statistically significant at $p<0.05: a$ ) bleomycin induced $v s$. control; $b$ ) bleomycin+EGCG $v s$. bleomycin induced.

The difference in the activities of lung SOD, CAT, GPx and GR in all the experimental and control rats is summarized in Table 3. Bleomycin induction to animals resulted in a significant decrease of enzyme activities when compared to control animals. EGCG treatment to bleomycin-induced animals significantly upturned the activities of these enzymes towards near normalcy.

Table 4 shows the level of GSH in lungs and plasma of control and experimental group of rats. Bleomycin-induced animals exhibited a significant lower level of GSH when compared to the control group. The GSH levels in plasma and in lungs were significantly elevated in bleomycin-induced rats treated with EGCG.

The levels of vitamin $\mathrm{C}$, vitamin $\mathrm{E}$ and vitamin $\mathrm{A}$ in the lung of control and experimental group of rats are presented in Fig. 1. A significant decrease in the levels was evident in bleomycin-induced animals when compared to control animals. The levels of these non-enzymic antioxidants were significantly increased in bleomycin-induced animals treated with EGCG.

The effect of EGCG on lung histology of control and experimental groups is depicted in Fig. 2. Section of the control
Table 4. Levels of Reduced Glutathione (GSH) in Plasma and Lungs of Control and Experimental Group of Rats

\begin{tabular}{lcc}
\hline \hline Experimental groups & $\begin{array}{c}\text { Lung GSH } \\
(\mathrm{mg} / 100 \mathrm{~g} \text { tissue })\end{array}$ & $\begin{array}{c}\text { Plasma GSH } \\
(\mathrm{mg} / \mathrm{dl})\end{array}$ \\
\hline Control & $20.37 \pm 1.67$ & $25.64 \pm 2.10$ \\
EGCG & $19.86 \pm 1.64$ & $25.59 \pm 2.12$ \\
Bleomycin & $13.94 \pm 1.32^{a)}$ & $16.37 \pm 1.58^{a)}$ \\
Bleomycin+EGCG & $18.13 \pm 1.54^{b)}$ & $23.56 \pm 2.07^{b)}$ \\
\hline
\end{tabular}

Values are given as mean \pm S.D. for groups of 6 rats each. Values are given statistically significant at $p<0.05 ; a$ ) bleomycin induced $v s$. normal control; $b$ ) bleomycin + EGCG $v s$. bleomycin induced.

group displayed normal structure with hardly any pathological changes (Fig. 2A). Figure 2C represents bleomycin-induced group that displays thickening of alveolar septa and excess collagen deposition with large number of leukocyte accumulation. Less collagen accumulation with somewhat clear alveolar space and less leukocyte accumulation was observed in bleomycin-induced animals treated with EGCG (Fig. 2D). Figure 2B depicts EGCG alone treated group that shows similar structure as found in control group.

Figure 3 represents Masson's trichrome staining performed in the fixed lung tissue sections of control and experimental group of animals. Bleomycin-induced group (Fig. 3C) showed increased collagen deposit as evident from increased blue colour staining when compared to control group (Fig. $3 \mathrm{~A})$. Treatment with EGCG to bleomycin-induced pulmonary fibrosis group resulted in marked reduction of collagen deposition in the lung tissues (Fig. 3D). Figure 3B represents EGCG alone-administered group that shows a similar pattern as observed in control group. 


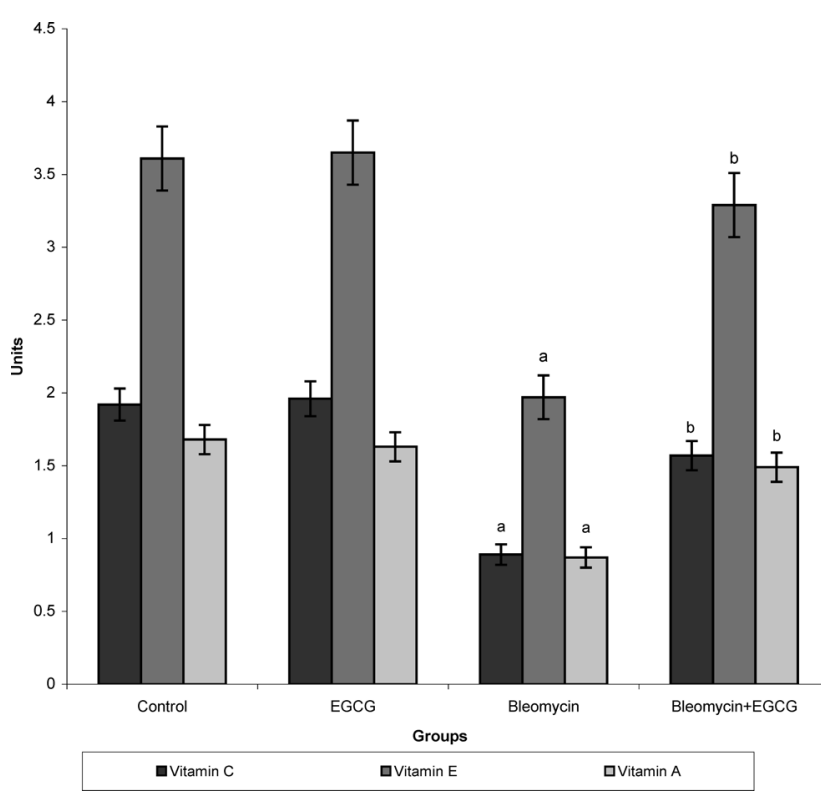

Fig. 1. Levels of Vitamin C, Vitamin E and Vitamin A in Lungs of Control and Experimental Group of Rats

Units are expressed as $\mu \mathrm{g} / \mathrm{mg}$ protein. Values are given as mean \pm S.D. for groups of 6 rats each. Values are given statistically significant at $p<0.05$ : ${ }^{a}$ bleomycin induced $v$ s. control; ${ }^{\mathrm{b}}$ bleomycin +EGCG $v$ s. bleomycin induced.
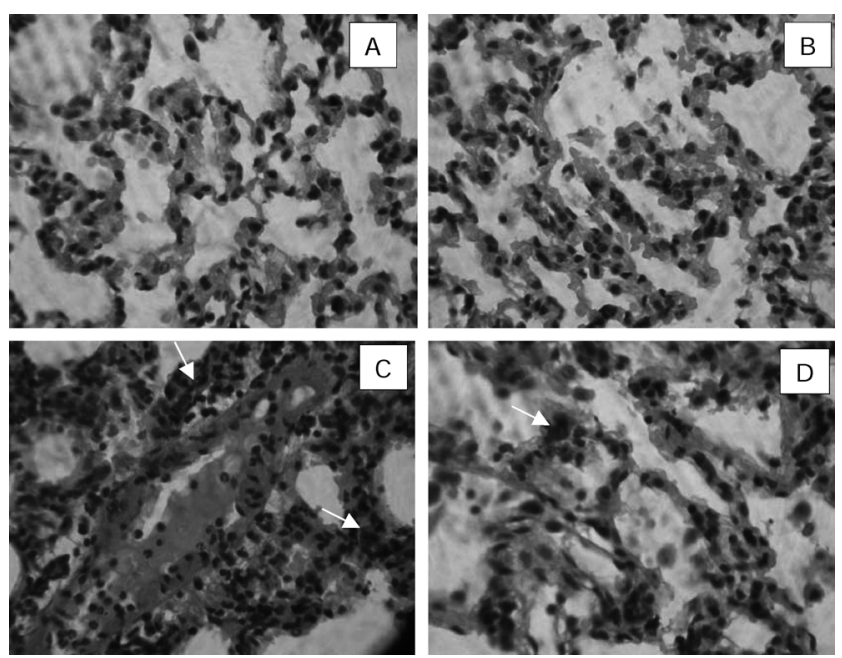

Fig. 2. Effect of EGCG on Lung Histology of Control and Bleomycin Induced Rats Stained with Hematoxylin \& Eosin (H \& E)

Sections were visualised under a light microscope at magnification $40 \times$. (A) Contro rats showing normal lung histology with clear alveolar spaces. (B) Represents contro rats treated with EGCG showing normal lung histology. (C) Bleomycin induced group displaying marked thickening of alveolar septa, collapse of alveolar spaces and large number of leukocytes accumulation in alveolar walls. (D) Lung of bleomycin +EGCG group showing less leukocyte accumulation with somewhat clear alveolar spaces.

\section{DISCUSSION}

Bleomycin, a highly effective antitumour agent, is known to produce pulmonary fibrosis that limits its clinical use. ${ }^{38)}$ Pulmonary fibrosis, idiopathic or otherwise, is commonly progressive and essentially an untreatable disease, with an increasingly fatal outcome. Tea polyphenol EGCG is a potent antioxidant and is an effective scavenger of free radicals. Though the preventive effect of green tea and EGCG against lipopolysaccharide induced inflammation and pulmonary fibrosis has been well documented, ${ }^{39)}$ the efficacy of EGCG

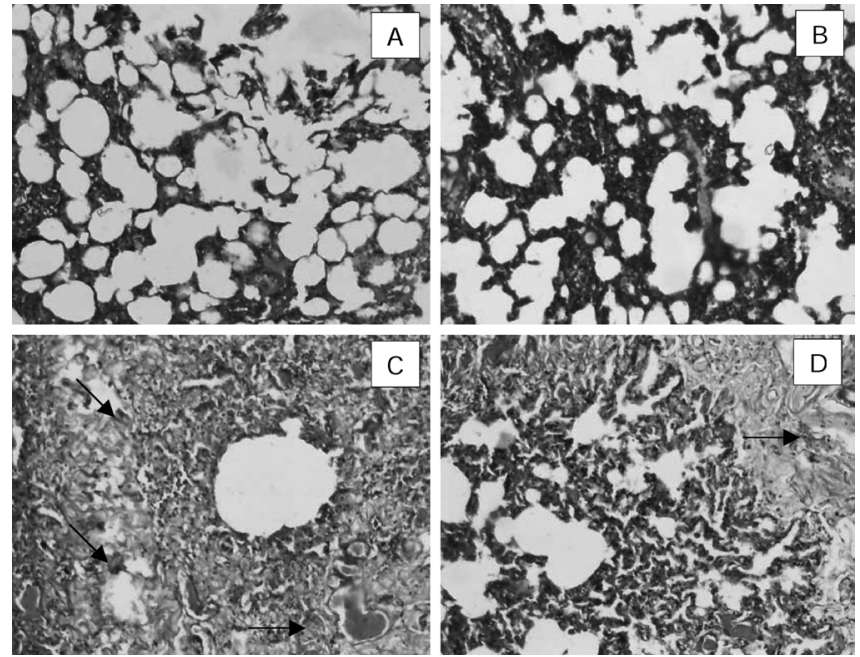

Fig. 3. Effect of EGCG on Collagen Accumulation in Lung Tissue Sections of Control and Bleomycin Induced Rats Stained with Masson's Trichrome

Masson's Trichrome is most useful to differentiate collagen from other fibres, particularly smooth muscle and elastin. The collagen in the following images is clearly seen as a dense bluish- tinged material, which is indicated by arrows, when stained with Masson's Trichrome (magnification 20×). (A) and (B) represents control and EGCG alone treated sections respectively that shows very little collagen deposit. (C) Bleomycin induced group shows intense blue colour staining throughout the section indicative of increased collagen accumulation and fibrosis. (D) Corresponds to bleomycin +EGCG treated group that shows less collagen deposit.

against bleomycin-induced pulmonary fibrosis, especially its effect on the antioxidant defense status during the fibrotic stage is still not known.

The fibrotic model (inducing pulmonary fibrosis through single intratracheal injection of bleomycin) adopted in this study was a well-established one. ${ }^{40,41)}$ The lung injury and fibrosis due to bleomycin induction was accessed by the measurements of rat body weights and hydroxyproline level of the lungs. The observed decrease in body weight with concomitant increase in hydroxyproline level in bleomycin-induced rats might be due to the acute injury to the lungs with subsequent development of fibrosis. The result of the present study was also consistent with the reported studies. ${ }^{42,43)}$ Pulmonary edema, which was evaluated by lung W/D ratio, is a potential marker for bleomycin-induced pulmonary fibrosis. ${ }^{44)}$ In our study, an increased lung W/D ratio was observed in bleomycin-induced animals that indicates severe fibrosis to lungs inflicted by bleomycin induction. Treatment of EGCG to bleomycin-induced animals not only increased the body weight but also decreased the hydroxyproline level and lung W/D ratio indicating its protective nature against fibrosis.

Any pathological state that leads to increased production and/or ineffective scavenging of reactive oxygen species may play a crucial role in determining tissue injury. ${ }^{45)}$ Bleomycin binds to iron $\left(\mathrm{Fe}^{2+}\right)$, undergoes redox cycling and catalyzes the formation of ROS that plays a major role in the progression of pulmonary fibrosis. ${ }^{46}$ Thus generated free radicals target biomacromolecules, such as DNA, protein and lipids, with the ultimate progression of lipid peroxidation. Increased LPO products in cells can result in cellular dehydration, whole cell deformity and cell death. Thus LPO is considered as the prime rationale that instigates lung injury. The most commonly used lipid peroxidation markers are TBARS and hydroperoxides. Induction of bleomycin, in our study, re- 
sulted in elevated levels of TBARS and hydroperoxides signifying the increased production of oxygen radicals. Similar reports of bleomycin-induced augmented LPO level has been reported. ${ }^{41)}$ Previous structure-activity studies indicated that the presence of a gallate (G)-ring and either a catechol (B)ring or a pyrogallol (B)-ring is important for the antioxidant activities of catechins. ${ }^{47)}$ Recent reports confirmed the inhibitory activity of EGCG on LPO in experimental animals. ${ }^{48)}$ The above reports were substantiated by our present study, where treatment with EGCG reduced LPO induced by bleomycin suggesting its antioxidant potential.

Since transition metals such as iron may play a central role in the process of lipid peroxidation during bleomycin induction, ${ }^{44)}$ reducing the availability of iron may lessen the extent of lipid peroxidation and culminate pulmonary fibrosis. In our study the decreased LPO production observed in EGCG treated group, might also be due to the iron chelating activity of EGCG ${ }^{49)}$ as green tea polyphenols have been shown to possess relatively potent metal chelating properties. ${ }^{21,50)}$ The potential metal chelating activity of EGCG is attributed to the gallate moiety present in its C-ring. ${ }^{51)}$ Thus the ability of EGCG to act as radical scavenger and chelate transitional metals such as iron, may be of major significance for treatment of bleomycin-induced pulmonary fibrosis.

Far and wide, it is accepted that the induction of antioxidant enzymes is a critical approach for protecting cells against a variety of endogenous and exogenous toxic compounds such as ROS. ${ }^{52)}$ SOD is an ubiquitous enzyme that catalyzes the dismutation of superoxide into oxygen and hydroperoxides thereby protecting the cells from detrimental superoxide anion. ${ }^{53)}$ CAT is a heme containing redox enzyme found in peroxisomes. It catalyses the conversion of hydrogen peroxide to water and molecular oxygen thus protecting the cells from oxidative damage. GPx, a selenium contaning enzyme, reduces lipid hydroperoxides to their corresponding alcohols and free hydrogen peroxide to water, eventually protecting the cells from oxidative damage. GR plays a significant role in regenerating GSH from GSSG (Oxidised glutathione), thus maintaining the balance between the redox couple. The equilibrium between these enzymes is important for the effective removal of free radicals and oxidative stress in intracellular organells. A notable descend in the activities of SOD, CAT, GPx and GR was observed in bleomycin-induced animals, which might be due to increased LPO and overproduction of ROS, which is in agreement with previous report. ${ }^{54)}$ The activities of these enzymes were maintained to near normal values upon treatment with EGCG. This may be due to the direct action of EGCG on superoxide, hydroxyl and alkoxyl radical coupled with its ability to attenuate LPO, which in turn reduces free radical generation and oxidative stress during pulmonary fibrosis.

Glutathione, a major non protein thiol normally present in millimolar concentrations in all cells, is known to protect the cellular system against the toxic effects of lipid peroxidation. ${ }^{55)}$ GSH reacts directly with ROS and electrophilic metabolites, protects essential thiol group from oxidation, and serves as a substrate for several enzymes including GPx. Decreased GSH levels indicates increased oxidative stress. Also, sustained oxidative challenge to the lung results in depletion of GSH and other antioxidants from the lungs. ${ }^{56)}$ Consistent with this concept, the downfall in the level of
GSH in bleomycin-induced animals might be due to enhanced utilization of this antioxidant for scavenging free radicals. EGCG treatment to bleomycin-induced animals increased the content of GSH in the plasma and lungs by alleviating the oxidative stress.

Vitamin E, the chief constituent in the membrane is regarded as a last line defense against membrane lipid peroxidation. ${ }^{57)}$ Thus its protection is by terminating the lipid peroxidation side chain rather than scavenging extracellular nonlipid radicals that initiate lipid peroxidation. Vitamin $\mathrm{C}$ is a naturally occurring free radical scavenger, which reduces free radical and lipid peroxidation sequence. It regenerates membrane-bound $\alpha$-tocopherol radical and removes the radical from the lipid to the aqueous phase. It also protects tissues from lipid peroxidation both in vivo and in vitro. ${ }^{58)}$ Vitamin $\mathrm{A}$ as an antioxidant is involved in counteracting free radicals and is known to help in the repair of damaged tissues. ${ }^{59)}$ At pharmacological doses, vitamin A is reported to assuage the oxidative stress mediated lipid peroxidation and membranes enriched with vitamin A are protected against oxidative stress in vivo and exhibit resistance to LPO induced in vitro. ${ }^{60)}$ The decline in the levels of vitamin $\mathrm{C}$, vitamin $\mathrm{E}$ and vitamin $\mathrm{A}$ in the lungs of bleomycin-induced rats might be related to insufficient antioxidant potential due to increased lipid peroxidation and oxidative stress. However, decreased activity of these vitamins observed in bleomycin-induced rats was significantly regulated by EGCG treatment, indicating modulation over oxidative stress.

Intratracheal administration of bleomycin to rodents is considered to thoroughly reproduce the histologic alterations that are found in human pulmonary fibrosis. In our experiments, we have observed marked distortion of the alveolar space with abnormal collagen deposits coupled with large number of leukocyte invasion in bleomycin-induced rats. These histological changes could be attributed to increased oxidative stress, which not only instigates inflammation but also leads to abnormal collagen production. ${ }^{61)}$ The rats treated with EGCG portrayed normal histology due to their antioxidant potency.

The results of this study suggest that intratracheal administration of bleomycin resulted in free radical production and pulmonary fibrosis. The role of EGCG as a free radical quencher and its task in preserving the pulmonary antioxidant status and membrane stability are apparent from our studies. Antioxidant treatment to pulmonary fibrosis is now considered an effective way to combat the disease. Thus to conclude, it can be stated that the EGCG due to its antioxidant activity could form an effective drug regimen for pulmonary fibrosis. Further studies on multiple mechanisms that devise the protective effect against pulmonary fibrosis and mechanism of action of EGCG are in progress.

\section{REFERENCES}

1) Cadenas E., Davies K. J., Free Radic. Biol. Med., 29, 222-230 (2000).

2) Halliwell B., Gutteridge J. M. C., "Free Radicals in Biology and Medicine," Clarendon Press Inc., Oxford, 2001.

3) Turrens J. F., Alexandre A., Lehninger A. L., Arch. Biochem. Biophys., 237, 408-414 (1985).

4) Sies H., Eur. J. Biochem., 215, 213-219 (1993).

5) Sies H., Exp. Physiol., 82, 291-295 (1997). 
6) Ates B., Yilmaz I., Geckil H., Iraz M., Birincioglu M., Fiskin K., $J$. Pineal Res., 37, 149-152 (2004).

7) Clark J. M., Lambertson C. J., Pharmacol. Rev., 23, 37-133 (1971).

8) Winterhalder R. C., Hirsch F. R., Kotantoulas G. K., Franklin W. A., Bunn P. A. Jr., Ann. Oncol., 15, 185-196 (2004).

9) Thannickal V. J., Toews G. B., White E. S., Lynch J. P. 3rd, Martinez F. J., Annu. Rev. Med., 55, 395- 417 (2004).

10) Garantziotis S., Steele M. P., Schwartz D. A., J. Clin. Invest., 114, 319-321 (2004)

11) Lasky J. A., Ortiz L. A., Am. J. Med. Sci., 322, 213-221 (2001).

12) Mapel D. W., Samet J. M., Coultas D. B., Chest, 110, 1058-1067 (1996).

13) Chen J., Stubbe J., Nat. Rev. Cancer, 5, 102-112 (2005).

14) Azambuja E., Fleck J. F., Batista R. G., Menna Barreto S. S., Pulm. Pharmacol. Ther., 18, 363-366 (2005).

15) Cantin A. M., Hubbard R. C., Crystal R. G., Am. Rev. Respir. Dis., 139, 370-372 (1989).

16) Kalayarasan S., Sriram N., Sudhandiran G., Life Sci., DOI: 10.1016/j.lfs.2008.03.018 (2008)

17) Atzori L., Chua F., Dunsmore S. E., Willis D., Barbarisi M., McAnulty R. J., Laurent G. J., Thorax, 59, 217-223 (2004).

18) Oury T. D., Thakker K., Menache M., Chang L. Y., Crapo J. D., Day B. J., Am. J. Respir. Cell Mol. Biol., 25, 164-169 (2001).

19) Giri S. N., Biring I., Nguyen T., Wang Q., Hyde D. M., Nitric Oxide, 7, 109-118 (2002).

20) Harborne J. B., "Nature Distribution and Function of Plant Flavonoids," ed. by Cody B., Middleton E., Harborne J. B., Alan Liss, New York, 1986, pp. 15-24.

21) Guo Q., Zhao B., Li M., Shen S., Xin W., Biochim. Biophys. Acta, 1304, 210-222 (1996).

22) Jin C. F., Shen S. R. Sr., Zhao B. L., J. Agric. Food Chem., 49, 60336038 (2001)

23) Rice-Evans C., Proc. Soc. Exp. Biol. Med., 220, 262-266 (1999).

24) Katiyar S. K., Mukhtar H., Int. J. Oncol., 8, 221—238 (1996).

25) Wang H. D., Yamaya M., Okinaga S., Jia Y. X., Kamanaka M., Takahashi H., Guo L. Y., Ohrui T., Sasaki H., Am. J. Respir. Crit. Care Med., 165, 406-411 (2002).

26) Ohkawa H., Ohishi N., Yagi K., Anal. Biochem., 95, 351-358 (1979).

27) Jiang Z. Y., Hunt J. V., Wolff S. P., Anal. Biochem., 202, 384-389 (1992).

28) Misra H. P., Fridovich I., J. Biol. Chem., 247, 3170-3175 (1972).

29) Takahara S., Hamilton H. B, Neel J. V., Kobara T. Y., Ogura Y., Nishimura E. T., J. Clin. Invest., 39, 610-619 (1960).

30) Rotruck J. T., Pope A. L., Ganther H. E., Swanson A. B., Hafeman D. G., Hoekstra W. G., Science, 179, 588-590 (1973).

31) Staal G. E., Visser J., Veeger C., Biochim. Biophys. Acta, 185, 39-48 (1969).

32) Ellman G. L., Arch. Biochem. Biophys., 82, 70-77 (1959).

33) Omaye S. T., Tumbull J. D., Sauberlich H. E., Methods Enzymol., 62, 3-11 (1979).
34) Desai I. D., Methods Enzymol., 105, 138-147 (1984).

35) Bayfield R. F., Cole E. R., Methods Enzymol., 67, 189-195 (1980).

36) Neuman R. E., Logan M. A., J. Biol. Chem., 186, 549-556 (1950).

37) Lowry O. H., Rosebrough N. J., Farr A. L., Randall R. J., J. Biol. Chem., 193, 265-275 (1951).

38) Keane M. P., Belperio J. A., Arenberg D. A., Burdick M. D., Xu Z. J., Xue Y. Y., Strieter R. M., J. Immunol., 163, 5686-5692 (1999).

39) Donà M., Dell'Aica I., Calabrese F., Benelli R., Morini M., Albini A., Garbisa S., J. Immunol., 170, 4335-4341 (2003).

40) Mutsaers S. E., Foster M. L., Chambers R. C., Laurent G. J., McAnulty R. J., Am. J. Respir. Cell Mol. Biol., 18, 611—619 (1998).

41) Sogut S., Ozyurt H., Armutcu F., Kart L., Iraz M., Akyol O., Ozen S., Kaplan S., Temel I., Yildirim Z., Eur. J. Pharmacol., 494, 213-220 (2004).

42) Izbicki G., Segel M. J., Christensen T. G., Conner M. W., Breuer R., Int. J. Exp. Pathol., 83, 111-119 (2002).

43) Mutsaers S. E., McAnulty R. J., Laurent G. J., Knight D. A., Drug Discov. Today Dis. Model, 1, 329-336 (2004).

44) Gong L. K., Li X. H., Wang H., Zhang L., Cai Y., Qi X. M., Liu L. L., Liu Y. Z., Wu X. F., Chen F. P., Huang C. G., Ren J., Biol. Pharm. Bull., 27, 634-640 (2004).

45) Halliwell B., Gutteridge J. M., Lancet, 1, 1396-1397 (1984).

46) Martin W. J. 2nd, Kachel D. L., J. Lab. Clin. Med., 110, 153-158 (1987).

47) Mukai K., Nagai S., Ohara K., Free Radic. Biol. Med., 39, 752-761 (2005).

48) Chen J. H., Tipoe G. L., Liong E. C., So H. S., Leung K. M., Tom W M., Fung P. C., Nanji A. A., Am. J. Clin. Nutr., 80, 742-751 (2004).

49) Afanas'ev I. B., Dorozhko A. I., Brodskii A. V., Kostyuk V. A., Potapovitch A. I., Biochem. Pharmacol., 38, 1763-1769 (1989).

50) Grinberg L. N., Newmark H., Kitrossky N., Rahamim E., Chevion M., Rachmilewitz E. A., Biochem. Pharmacol., 54, 973-978 (1997).

51) Kumamoto M., Sonda T., Nagayama K., Tabata M., Biosci. Biotechnol. Biochem., 65, 126-132 (2001).

52) Sen C. K., Indian J. Physiol. Pharmacol., 39, 177-196 (1995).

53) McCrod J. M., Keele B. B. Jr., Fridovich I., Proc. Natl. Acad. Sci. U.S.A., 68, 1024-1027 (1971).

54) Iraz M., Erdogan H., Kotuk M., Yağmurca M., Kilic T., Ermis H., Fadillioğlu E., Yildirim Z., Pharmcol. Res., 53, 310-316 (2006).

55) Lu S. C., FASEB J., 13, 1169-1183 (1999).

56) Meister A., Cancer Res., 54, 1969s-1975s (1994).

57) Gerster H., Eur. J. Clin. Nutr., 49, 155-168 (1995).

58) Choi S. W., Benzie I. F., Collins A. R., Hannigan B. M., Strain J. J., Mutat. Res., 551, 109-117 (2004).

59) Serbecic N., Ehmann A. K., Beutelspacher S. C., Ophthalmology, 102, 607-613 (2005).

60) Britton J. A., Westhoff C., Howe G., Gammon M. D., Cancer Causes Control, 11, 389-401 (2000).

61) Grande N. R., Peao M. N. D., de sa C. M., Aguas A. P., Scanning Microsc., 12, 487-494 (1998). 